\title{
Statistic of the winding of geodesics on a Riemann surface with finite area and constant negative curvature
}

Nathanaël Enriquez and Yves Le Jan

Abstract. In this paper we show that the windings of geodesics around the cusps of a Riemann surface of finite area, behave asymptotically as independent Cauchy variables.

\section{Introduction.}

In this paper we show that the windings of geodesics around the cusps of a Riemann surface of finite area, behave asymptotically as independent Cauchy variables. Results of this type were originally given for Brownian paths. The original proof of [16] for the winding of planar Brownian motion around the origin was analytic. This theory was developed in many works including [1], [2], [14], [9] and [12] using excursion theory and geometric ideas. The idea that such a result might hold for geodesics is suggested by the central limit theorem of Ratner [13] and Sinaï, and the logarithm iterated law discovered by Sullivan [17]. Using coding theory a proof is given in [3] and [4] for modular surfaces. In the note [10], it was briefly shown that this result could be extended to arbitrary Riemann surfaces, by a simple argument that 
reduced the problem to the Brownian case. However, in these works, the contribution $e_{t}^{i}$ of each cusp $C_{i}$ was not identified. The asymptotic was actually obtained for linear combinations $\sum \lambda_{i} e_{t}^{i}$ under the condition that $\sum \lambda_{i}=0$. We show that this condition is unnecessary, using the relation between the Brownian motion on the stable foliation and the geodesic flow which was obtained in [11]. It is reasonable to think that the constant curvature assumption could be relaxed as in [7], [8].

\section{Presentation of the result.}

Let $M$ be a surface of constant negative curvature with finite area, represented as the quotient of the hyperbolic plane $\mathrm{H}$, under the action of a Fuchsian group $\Gamma$.

The well known model of the hyperbolic plane, using the upper half-plane $\mathbb{C}^{+}$with the metric $d l^{2}=\left(d x^{2}+d y^{2}\right) / y^{2}(y>0)$, can be transformed into the model of the open unit disc via a conformal map, the metric being then

$$
d l^{2}=\frac{d x^{2}+d y^{2}}{\left(1-x^{2}-y^{2}\right)^{2}}, \quad x^{2}+y^{2}<1 .
$$

In the representation of the disc, there exists a polygon (whose edges are geodesics) which is a fundamental domain for $\Gamma$. There comes out some invariants of the group, (independent from the choice of the system of generator) like its genus $g$ and the multiplicity of the vertices of the polygon. $M$ in our case, will be the union of a compact part and of $n$ cusps $C_{1}, C_{2}, \ldots, C_{n}$, a cusp being the region of the polygon limited by two geodesics going at infinity to the same point of the boundary of the hyperbolic plane (though it is non compact, this region remains of finite area).

Let $m$ be the normalized Liouville measure on the unit tangent bundle $T^{1} M$. Functions on $T^{1} M$ can be viewed as random variables on the probability space $\left(T^{1} M, \mathcal{B}, m\right)(\mathcal{B}$ denoting the Borel $\sigma$-field on $\left.T^{1} M\right)$.

We denote by $\theta_{t}$ the geodesic flow on $T^{1} M$, which preserves $m$ and is known to be ergodic [5].

Let $\omega$ be a 1 -form on $M$ : we assume that $d \omega$ vanishes in a neighbourhood $U_{i}$ of each cusp $C_{i}$. Let $\lambda_{i}$ denote the residue of $\omega$ at $C_{i}$ (which is the integral of $\omega$ along a loop around $C_{i}$, included in $U_{i}$, which doesn't depend on the loop as far as this form is locally closed). 
If $\xi=(q, v), q \in M, v \in T_{q}^{1} M$, set $\theta_{t}(\xi)=\left(q_{t}, v_{t}\right)$, and

$$
e_{t}^{i}(\xi)=\int_{0}^{t}\left\langle\omega\left(q_{s}\right), v_{s}\right\rangle \mathbf{1}_{U_{i}}\left(q_{s}\right) d s
$$

(If $\lambda_{i}$ does not vanish, $e_{t}^{i}$ describes the winding of the geodesic in $U_{i}$ ).

We prove the following:

Theorem 1. The joint distribution of $\left(e_{t}^{1} / t, e_{t}^{2} / t, \ldots, e_{t}^{n} / t\right)$ converges in law towards the product of n Cauchy distributions of parameter $\left|\lambda_{i}\right| /$ $|M|$ where $|M|$ denotes the area of $M$.

REMARKS. If $\hat{\omega}$ is another form, closed near the cusps, with the same residues, the theorem applied to $\omega-\hat{\omega}$ implies that $\left(\hat{e}_{t}^{i}-e_{t}^{i}\right) / t$ converges to $0 m$ almost surely.

If $d \omega=0$ on $M, \sum \lambda_{i}$ vanishes. Since we assume only that $d \omega$ vanishes near the cusps, the residues can take arbitrary values. Therefore our theorem describes the winding of the geodesics around each cusp. This was not achieved in [4] and [10] where only the case of closed forms was treated.

Finally from the theorem we get the independence of the limit from the choice of the neighbourhoods.

If $\left\{\tilde{e}_{t}^{i}: 1 \leq i \leq n\right\}$ is defined using a different system of neighbourhoods $\left\{\tilde{U}_{i}: 1 \leq i \leq n\right\},\left(\tilde{e}_{t}^{i}-e_{t}^{i}\right) / t$ converges to $0 m$ almost surely.

This comes from the lemma we shall use in the following:

Lemma 1. If $\omega$ is a 1 -form, $\phi$ is a $C^{\infty}$-function of compact support in $M$, then

$$
\lim _{t \rightarrow+\infty} \frac{1}{t} \int_{0}^{t}\left\langle\omega\left(q_{s}\right), v_{s}\right\rangle \phi\left(q_{s}\right) d s \longrightarrow 0, \quad \text { almost surely }
$$

ProOF. This comes from the ergodic theorem, as far as

$$
\int \omega(q, v) \phi(q) d m(q, v)=0
$$

because the transformation $\sigma:(q, v) \longmapsto(q,-v)$ changes the sign of the integrated function, and $m$ is $\sigma$-invariant. 
Notations. H will be represented by the complex upper half-plane $\{z=x+i y: y>0\}$. We shall identify $T^{1} H$ and $P S L_{2}(\mathbb{R})$ using the relations

$$
q=\frac{a i+b}{c i+d} \quad \text { and } \quad v=\frac{i}{(c i+d)^{2}} .
$$

$\Gamma$ appears as a subgroup of $P S L_{2}(\mathbb{R})$. It is well known that $T^{1} M$ can be identified with $\Gamma \backslash P S L_{2}(\mathbb{R})$, in such a way that $\theta_{t}(\xi)$ can be written $\xi \theta_{t}$, if we set

$$
\theta_{t}=\left(\begin{array}{cc}
e^{t / 2} & 0 \\
0 & e^{-t / 2}
\end{array}\right)
$$

Similarly the right actions of the 1-parameter subgroups

$$
\theta_{t}^{+}=\left(\begin{array}{cc}
1 & t \\
0 & 1
\end{array}\right) \quad \text { and } \quad \theta_{t}^{-}=\left(\begin{array}{cc}
1 & 0 \\
t & 1
\end{array}\right)
$$

define the horocyclic flows on $T^{1} M$.

We can define the operators of derivation $L_{0}, L_{+}$and $L_{-}$on $C^{1}$ functions of $T^{1} M$ by

$$
\begin{aligned}
& L_{0} f(\xi)=\left.\frac{d}{d s}\right|_{s=0} f\left(\xi \theta_{s}\right), \\
& L_{+} f(\xi)=\left.\frac{d}{d s}\right|_{s=0} f\left(\xi \theta_{s}^{+}\right), \\
& L_{-} f(\xi)=\left.\frac{d}{d s}\right|_{s=0} f\left(\xi \theta_{s}^{-}\right) .
\end{aligned}
$$

For $\alpha>0$ and $f \in L^{2}(m)$, we can also define a resolvent operator

$$
R_{\alpha} f(\xi)=\int_{0}^{\infty} e^{-\alpha t} f\left(\xi \theta_{t}\right) d t
$$

We introduce the matrix $T_{z}$

$$
T_{z}=\frac{1}{\sqrt{y}}\left(\begin{array}{cc}
y & x \\
0 & 1
\end{array}\right)
$$

and we recall the formulas $T_{z} T_{z^{\prime}}=T_{x+y z^{\prime}}$ and the decomposition of $T_{z}$ in terms of the geodesic and horocyclic operators: $T_{x+i y}=\theta_{x}^{+} \theta_{\log y}=$ $\theta_{\log y} \theta_{x / y}^{+}$. We deduce from there the commutation formulas

$$
\theta_{-\log y} \theta_{x}^{+} \theta_{\log y}=\theta_{x / y}^{+} \quad \text { and } \quad \theta_{-\log y} \theta_{x}^{-} \theta_{\log y}=\theta_{x y}^{-} .
$$


From these commutation formulas we deduce the following equalities which will be useful especially in the proof of the convergence of some $R_{\alpha}$-type integrals

$$
\begin{gathered}
L_{+}^{k}\left(\phi\left(\xi \theta_{s}\right)\right)=e^{-k s}\left(L_{+}^{k} \phi\right)\left(\xi \theta_{s}\right), \\
L_{-}^{k}\left(\phi\left(\xi \theta_{s}\right)\right)=e^{k s}\left(L_{-}^{k} \phi\right)\left(\xi \theta_{s}\right), \\
\text { for all } \phi \in C^{\infty}\left(T^{1} M\right) \text { and } k .
\end{gathered}
$$

The influence of the geodesic and horocyclic operators is described by the following formulas

$$
T_{z} \theta_{s}^{+}=T_{x+y s+i y} \quad \text { and } \quad T_{z} \theta_{s}=T_{x+i y e^{s}} .
$$

The foliation $\left\{\xi T_{z}, z \in H\right\}$, describes all the matrices we can obtain from $\xi$ by the action of the geodesic and horocyclic flows.

Lastly, we shall denote the rotations of $P S L_{2}(\mathbb{R})$ by

$$
K_{t}=\left(\begin{array}{cc}
\cos \left(\frac{t}{2}\right) & \sin \left(\frac{t}{2}\right) \\
-\sin \left(\frac{t}{2}\right) & \cos \left(\frac{t}{2}\right)
\end{array}\right)
$$

\section{Reduction of the problem.}

We shall denote by $p$ the canonical projection of $\mathrm{H}$ on $M$ and by $\pi$ the canonical projection of $T^{1} M$ on $M$.

Each cusp $C_{i}$ is represented by a $\Gamma$-orbit on the boundary of $\mathrm{H}$, i.e. the projective line $\mathbb{R} \cup \infty$. Picking up an element $\overline{C_{i}}$ in that orbit we can choose $\gamma_{i}$ in $P S L_{2}(\mathbb{R})$ such that $\gamma_{i}^{-1}(\infty)=\overline{C_{i}}$. The subgroup of $\Gamma$ which consists of the elements which fix $\overline{C_{i}}$, can be written $\left\{\gamma_{i}^{-1} \theta_{n X_{i}}^{+} \gamma_{i}, n \in \mathbb{Z}\right\}$ where $X_{i}$ is a positive number independent of the choice of $\overline{C_{i}}$ and $\gamma_{i}$.

We define a fundamental domain $\mathcal{F}_{i}$ of $\Gamma$ contained in $\left\{\gamma_{i}^{-1} z: 0 \leq\right.$ $\left.x \leq X_{i}\right\}$, and containing $R_{h_{i} / 4}=\left\{\gamma_{i}^{-1} z: 0 \leq x \leq X_{i}, y \geq h_{i} / 4\right\}$ for some positive $h_{i}$. Choosing $h_{i}$ large enough, we can take $U_{i}=p\left(R_{h_{i} / 4}\right)$ and assume the $U_{i}$ 's are disjoint. We shall denote $p\left(R_{h_{i} / 3}\right)$ by $V_{i}$ and $p\left(R_{h_{i}}\right)$ by $W_{i}$.

Lastly we denote $U=\cup_{i=1}^{n} U_{i}, V=\cup_{i=1}^{n} V_{i}$, and $W=\cup_{i=1}^{n} W_{i}$. 
Let $u$ be a $C^{\infty}$ function on $\mathbb{R}^{+}$, such that $u=0$ on $[0,1 / 4]$, and $u=1$ on $[1 / 3,+\infty]$.

Let $s_{i}$ denote the section of $p$ relative to $\mathcal{F}_{i}$. There is a 1 -form $\eta$ on $M$ that vanishes outside $U=\cup_{i=1}^{n} U_{i}$ and represented in $U_{i}$ by

$$
s_{i}^{\star} \gamma_{i}^{\star}\left(\frac{\lambda_{i}}{X_{i}} d x u\left(\frac{y}{h_{i}}\right)\right)
$$

on $U_{i}$.

Inside $W_{i}, \omega-\eta$ is a closed form with 0-residue. Therefore (since $W_{i}$ is isomorphic to a disc minus a point), it is exact. Let $F_{i}$ be a smooth function on $W_{i}$ such that $\omega-\eta=d F_{i}$ on $W_{i} . \quad F_{i}$ will be extended into a smooth function vanishing outside $V_{i}$. Then the 1-form $\omega_{0}=\omega-\eta-\sum_{i=1}^{n} d F_{i}$, vanishes on $W$.

Note that

$$
\begin{aligned}
\frac{e_{t}^{i}}{t}= & \frac{1}{t} \int_{0}^{t}\left\langle\omega_{0}\left(q_{s}\right), v_{s}\right\rangle \mathbf{1}_{U_{i}}\left(q_{s}\right) d s \\
& +\frac{1}{t} \sum_{j=1}^{n}\left(F_{j}\left(\xi \theta_{t}\right)-F_{j}(\xi)\right)+\frac{1}{t} \int_{0}^{t}\left\langle\eta\left(q_{s}\right), v_{s}\right\rangle d s .
\end{aligned}
$$

Since $\theta_{t}$ preserves $m, F_{i}\left(\xi \theta_{t}\right)$ is a stationary process, so the middle term converges to 0 in probability (without any assumptions on the integrability of $F$ ).

The first term converges to $0 \mathrm{~m}$ p.s. by application of the ergodic theorem: indeed $\left\langle\omega_{0}(q), v\right\rangle \mathbf{1}_{U_{i}}(q)$ is an integrable function $T^{1} M$ since it vanishes everywhere except on the compact set $\left({ }^{c} W \cap U\right) \times S^{1}$. Moreover the mean value of this function is 0 . Indeed, the transformation $\sigma$ : $(q, v) \longmapsto(q,-v)$ changes the sign of the function, and $m$ is $\sigma$-invariant. written

Setting $\phi(\xi)=\langle\eta(q), v\rangle$, where $\xi=(q, v)$, the third term can be

$$
\frac{1}{t} \int_{0}^{t} \phi\left(\xi \theta_{s}\right) d s .
$$

Since the residues $\lambda_{i}$ are arbitrary, the theorem can be reduced to the

Proposition 1. The law of

$$
\frac{1}{t} \int_{0}^{t} \phi\left(\xi \theta_{s}\right) d s
$$


converges in law towards a Cauchy distribution of parameter

$$
\sum_{i=1}^{n} \frac{\left|\lambda_{i}\right|}{|M|}
$$

\section{Expression of $\phi$.}

We shall first introduce a fundamental domain for $T^{1} M$, as it was already for $M$ :

$\hat{\mathcal{F}}_{j}=\left\{g \in P S L_{2}(\mathbb{R}): g(i) \in \mathcal{F}_{j}\right\}$, is a fundamental domain for the left action of $\Gamma$ on $P S L_{2}(\mathbb{R})$. It is possible to characterize any element $\xi$ of $T^{1} M$ by its representative $g_{i}(\xi)$ in $\hat{\mathcal{F}}_{i}$.

We can define the Iwasawa coordinates $z_{i}(\xi)=x_{i}(\xi)+i y_{i}(\xi)$ and $\theta_{i}(\xi)$ by the equation $\gamma_{i} g_{i}(\xi)=T_{z_{i}(\xi)} K_{\theta_{i}(\xi)}$.

Note that if

$$
T_{z} K_{\theta}=\left(\begin{array}{ll}
a & b \\
c & d
\end{array}\right), \quad y=\frac{1}{c^{2}+d^{2}} \quad \text { and } \quad \sin \theta=\frac{-2 c d}{c^{2}+d^{2}}
$$

It can be easily seen that $\theta_{i}(\xi)$ and $y_{i}(\xi) / h_{i}$ have a geometrical interpretation: of $W_{i}$

- $y_{i} / h_{i}$ is the exponential of the distance from $\pi(\xi)$ to the boundary

- $\theta_{i}$ is the angle between the geodesic going from $\pi(\xi)$ to $C_{i}$ and the geodesic $\left\{\xi \theta_{t}, t \geq 0\right\}$.

We can deduce, from the definition of $\eta$ the following expression of $\phi$ in the $y_{i}, \theta_{i}$ coordinates

$$
\phi(\xi)=-\sum_{i=1}^{n} \frac{\lambda_{i}}{X_{i}} u\left(\frac{y_{i}(\xi)}{h_{i}}\right) y_{i} \sin \theta_{i}(\xi) \mathbf{1}_{U_{i}}(\pi(\xi))
$$

for all $\xi \in T^{1} M$ (it is worth remarking that although $\phi$ is a function on $T^{1} M$, it depends only on 2 dimensions). It is useful to give the expression of the differential operators $L_{0}$ and $L_{+}$in terms of $y_{i}$ and $\theta_{i}$ : 
Lemma 2. Let $F$ be a function on $U_{i}$, of the form $G\left(y_{i}(\xi), \theta_{i}(\xi)\right)$. Then

$$
\begin{gathered}
\left.L_{0} F(\xi)\right|_{U_{i}}=y_{i} \cos \theta_{i} \frac{\partial G}{\partial y_{i}}+\sin \theta_{i} \frac{\partial G}{\partial \theta_{i}} \\
\left.L_{+} F(\xi)\right|_{U_{i}}=y_{i} \sin \theta_{i} \frac{\partial G}{\partial y_{i}}+\left(1-\cos \theta_{i}\right) \frac{\partial G}{\partial \theta_{i}}
\end{gathered}
$$

Let us finally introduce the function

$$
\tilde{\phi}^{\prime}(\xi)=\sum_{i=1}^{n} \frac{\lambda_{i}}{X_{i}} u\left(\frac{y_{i}(\xi)}{h_{i}}\right) y_{i} \cos \theta_{i}(\xi) \mathbf{1}_{U_{i}}(\pi(\xi)) .
$$

The interest of this function lies in the following:

Lemma 3. Let $\omega_{\xi}^{\prime}$ be the 1-form on $H$ defined by the equation

$$
\omega_{\xi}^{\prime}(z)=\tilde{\phi}^{\prime}\left(\xi T_{z}\right) \frac{d x}{y}+\phi\left(\xi T_{z}\right) \frac{d y}{y}
$$

and let $j_{\xi}$ be the application from $H$ to $M$ which maps $z$ onto $\pi\left(\xi T_{z}\right)$. Then we get

$$
\omega_{\xi}^{\prime}=j_{\xi}^{\star} \eta
$$

Proof. The proof is just a matter of change of variables.

\section{A differential form.}

To follow the spirit of the proofs given in [10] and [11], we have to introduce closed forms. We first notice that since $\theta_{s}=T_{i e^{s}}$,

$$
\int_{0}^{t} \phi\left(\xi \theta_{s}\right) d s=\int_{1}^{e^{t}} \phi\left(\xi T_{i y}\right) \frac{d y}{y} .
$$

We shall introduce a function $\tilde{\phi}$ such that

$$
\omega^{\xi}=\phi\left(\xi T_{z}\right) \frac{d y}{y}+\tilde{\phi}\left(\xi T_{z}\right) \frac{d x}{y}
$$


is a closed form on $\mathrm{H}$, so that we will get

$$
\int_{0}^{t} \phi\left(\xi \theta_{s}\right) d s=\int_{i}^{i e^{t}} \omega^{\xi}
$$

(the second integral being independent of the path from $i$ to $i e^{t}$ ).

$\tilde{\phi}(\xi)$ will be defined by the integral

$$
-\int_{0}^{\infty} e^{-t} L_{+} \phi\left(\xi \theta_{t}\right) d t
$$

Its convergence will be proved using the following lemma:

Lemma 4. Let $\chi$ be a locally bounded function on $\Gamma \backslash S L_{2}(\mathbb{R})$ such that for some positive constant $P, \chi(\xi)$ is bounded by

$$
P y_{i}\left(1-\cos \theta_{i}\right)=P \frac{2 d_{i}^{2}}{\left(c_{i}^{2}+d_{i}^{2}\right)^{2}}
$$

in $V_{i}$, for every $i$, where $a_{i}, b_{i}, c_{i}, d_{i}$ denote the matrix coefficients of the matrix $\gamma_{i} g_{i}(\xi)$, and $y_{i}$ and $\theta_{i}$ its Iwasawa coordinates. Then

$$
\int_{0}^{+\infty} e^{-s} \chi\left(\xi \theta_{s}\right) d s
$$

converges uniformly in $\xi$.

Proof. As

$$
\int_{t_{0}}^{+\infty} e^{-s}\left|\chi\left(\xi \theta_{s}\right)\right| d t=e^{-t_{0}} \int_{0}^{+\infty} e^{-s}\left|\chi\left(\xi \theta_{t_{0}+s}\right)\right| d s,
$$

for all $t_{0} \in \mathbb{R}$, it is enough to get an upper bound of $\int_{0}^{+\infty} e^{-t}|\chi|\left(\xi \theta_{t}\right) d t$, independent of $\xi$ (the right integral being the value of this function for $\left.\xi \theta_{t_{0}}\right)$.

Outside $V,|\chi|$ is bounded so that the contribution of the part of the geodesic contained in ${ }^{c} V$ is uniformly bounded.

Hence it is enough to show that

$$
\sum_{i=1}^{n} \sum_{j \in \mathbb{N}} \int_{u_{i}^{j}}^{v_{i}^{j}} e^{-s}|\chi|\left(\xi \theta_{s}\right) d s
$$


is uniformly bounded where the disjoint intervals $\left[u_{i}^{j}, v_{i}^{j}\right]$ are defined by recursion as follows: $u_{i}^{j}$ denotes the first time after $v_{i}^{j-1}$ (or 0 if $j=0$ ) where the geodesic enters $W_{i}$ and $v_{i}^{j}$ the next exit time of $W_{i}$.

We will in fact majorize the contribution of each interval of excursion $\left[u_{i}^{j}, v_{i}^{j}\right]$ by the contribution of an asymmetric excursion $\left[u_{i}^{j}, u_{i}^{j}+s_{i}^{j}\right]$ such that $s_{i}^{j}$ is bounded below by a positive number and the geodesic between $u_{i}^{j}$ and $u_{i}^{j}+s_{i}^{j}$ lies in $V_{i}$.

Let us denote by $\xi_{i}^{j}$ the matrix $\gamma_{i} \xi_{u_{i}^{j}}$ and

$$
\xi_{i}^{j}=\left(\begin{array}{cc}
a_{i}^{j} & b_{i}^{j} \\
c_{i}^{j} & d_{i}^{j}
\end{array}\right)
$$

We get $1 /\left(c_{i}^{j^{2}}+d_{i}^{j^{2}}\right)=h_{i}$.

Let us show that $s_{i}^{j}=\log \left(2 /\left(c_{i}^{j^{2}} h_{i}\right)\right)$ satisfies the required properties.

First

$$
\frac{2}{c_{i}^{j^{2}} h_{i}} \geq \frac{2}{\left(c_{i}^{j^{2}}+d_{i}^{j^{2}}\right) h_{i}}=2
$$

thus $s_{i}^{j}>\log 2$. Second

$$
c_{i}^{j^{2}} d_{i}^{j^{2}} h_{i}^{2} \leq \frac{h_{i}^{2}\left(c_{i}^{j^{2}}+d_{i}^{j^{2}}\right)^{2}}{4}=\frac{1}{4}<2,
$$

so that

$$
\frac{h_{i}}{3}<\frac{1}{c_{i}^{j^{2}} e^{s_{i}^{j}}+d_{i}^{j^{2}} e^{-s_{i}^{j}}}=\frac{h_{i}}{2+\frac{c_{i}^{j^{2}} d_{i}^{j^{2}} h_{i}^{2}}{2}}<h_{i} .
$$

All the conditions concerning $s_{i}^{j}$ are hence satisfied.

We are going now to estimate the contribution of the $j^{\text {th }}$ passage of the geodesic in the neighbourhood of $C_{i}$, by

$$
\int_{u_{i}^{j}}^{u_{i}^{j}+s_{i}^{j}} e^{-s}|\chi|\left(\xi \theta_{s}\right) d s
$$

for which we are going to prove that it is the term of a convergent serie.

$$
\int_{u_{i}^{j}}^{u_{i}^{j}+s_{i}^{j}} e^{-s}|\chi|\left(\xi \theta_{s}\right) d s=e^{-u_{i}^{j}} \int_{0}^{s_{i}^{j}} e^{-s}|\chi|\left(\xi_{i}^{j} \theta_{s}\right) d s,
$$


with the above notation concerning the matrix $\xi_{i}^{j}$. We first notice that the minoration of $s_{i}^{j}$ by $\log 2$, gives $u_{i}^{j}>(j-1) \log 2$, so that

$$
e^{-u_{i}^{j}}<\frac{1}{2^{j-1}}
$$

Moreover,

$$
\begin{aligned}
\left|\int_{0}^{s_{i}^{j}} e^{-s}\right| \chi\left|\left(\xi_{i}^{j} \theta_{s}\right) d s\right| & \leq P \int_{0}^{\log \left(2 /\left(h c_{i}^{j^{2}}\right)\right)} e^{-s} \frac{c_{i}^{j^{2}} e^{3 s}}{\left(d_{i}^{j^{2}}+c_{i}^{j^{2}} e^{2 s}\right)^{2}} d s \\
& =P \int_{1}^{2 /\left(h c_{i}^{j^{2}}\right)} \frac{c_{i}^{j^{2}} x}{\left(d_{i}^{j^{2}}+c_{i}^{j^{2}} x^{2}\right)^{2}} d x \\
& =P\left[\frac{1}{\left(d_{i}^{j^{2}}+c_{i}^{j^{2}} x^{2}\right)}\right]_{1}^{2 /\left(h c_{i}^{j^{2}}\right)} \\
& \leq \frac{P}{2} \frac{1}{\left(d_{i}^{j}\right)^{2}+\left(c_{i}^{j}\right)^{2}} \\
& =\frac{P h}{2} .
\end{aligned}
$$

So the contribution of the $j^{\text {th }}$ passage is less than $M_{i} h / 2^{j}$, which is the term of a convergent serie. The lemma is proved.

Lemma 5. The function $\tilde{\phi}=-R_{1} L_{+} \phi$ is continuous.

ProOF. In each $V_{i}$ we have an explicit formula for $\phi$

$$
\phi(\xi)=-\frac{\lambda_{i}}{X_{i}} y_{i} \sin \theta_{i}
$$

Lemma 1 yields

$$
L_{+} \phi(\xi)=\frac{\lambda_{i}}{X_{i}} y_{i}\left(\cos \theta_{i}-1\right)\left(2 \cos \theta_{i}+1\right) \text {. }
$$

So $L_{+} \phi$ satifies the conditions of previous lemma, which ends the proof.

\section{Lemma 6.}

1) $L_{0} \tilde{\phi}$ and $L_{+} \tilde{\phi}$ are well defined and continuous, 
2) $\omega^{\xi}$ is a closed form with $C^{1}$ coefficients.

Proof. 1) For $L_{0} \tilde{\phi}$, we have to prove the uniform convergence in $\xi$ of

$$
\int_{0}^{\infty} e^{-s} L_{0} L_{+} \phi\left(\xi_{i} \theta_{s}\right) d s
$$

We just have to check the assumptions of Lemma 4. But using the formulas of Lemma 2, we get when $\pi(\xi) \in V_{i}$

$$
L_{0} L_{+} \phi(\xi)=\frac{2 \lambda_{i}}{X_{i}} y_{i}\left(1-\cos \theta_{i}\right)\left(1-4 \cos \theta_{i}-6 \cos ^{2} \theta_{i}\right)
$$

This function satisfies the assumptions of Lemma 4.

For $L_{+} \tilde{\phi}$, note that

$$
L_{+}\left(\int_{0}^{T} e^{-s} L_{+} \phi\left(\xi \theta_{s}\right) d s\right)=\int_{0}^{T} e^{-s} L_{+}\left(L_{+} \phi\left(\xi \theta_{s}\right)\right) d s
$$

To prove the uniform convergence in $\xi$ of the last integral when $T$ goes to $\infty$, we first note that

$$
L_{+}\left(L_{+} \phi\left(\xi \theta_{s}\right)\right)=e^{-s} L_{+}^{2} \phi\left(\xi \theta_{s}\right)
$$

An easy calculation yields

$$
L_{+}^{2} \phi(\xi)=\frac{6 \lambda_{i}}{X_{i}} y_{i} \sin \theta_{i} \cos \theta_{i}\left(\cos \theta_{i}-1\right),
$$

so we can conclude by Lemma 4 .

2) It is easy to check [11] that

$$
d \omega^{\xi}=\left(-L_{+} \phi+L_{0} \tilde{\phi}-\tilde{\phi}\right) \frac{d x \wedge d y}{y^{2}}
$$

and that the parenthesis vanishes by definition of $\tilde{\phi}$. 


\section{Geodesic flow and Brownian motion.}

We are going to show the relation between the integral of $\phi$ along the flow between 0 and $t$, which is equal to $\int_{i}^{i e^{t}} \omega^{\xi}$, and the integral along the Brownian path on $H$ starting at $i$.

Let us define the Brownian motion by the equations:

$$
\begin{aligned}
d x_{t} & =\sqrt{2} y_{t} d W_{t}^{(1)}, & x_{0} & =0, \\
d y_{t} & =\sqrt{2} y_{t} d W_{t}^{(2)}, & y_{0} & =1,
\end{aligned}
$$

where $W_{t}^{(1)}$ and $W_{t}^{(2)}$ are two real independent Brownian motions. The generator of the process so defined is

$$
y^{2}\left(\frac{\partial^{2}}{\partial x^{2}}+\frac{\partial^{2}}{\partial y^{2}}\right)
$$

(the explanation of the choice of this normalization will appear in Lemma 10). We shall denote $z_{t}=x_{t}+i y_{t}$. sense).

N.B. $\xi T_{z_{t}}$ is a Brownian motion on the leaf $\xi T_{z}$ (in the matricial

The relation between both flows is given in the following lemma:

\section{Theorem 2.}

$$
\begin{aligned}
\lim _{t \rightarrow+\infty} \int m(d \xi) \exp \left(\frac{i}{t} \int_{i}^{i e^{t}} \omega^{\xi}\right) & \\
& =\lim _{t \rightarrow+\infty} E\left[\int m(d \xi) \exp \left(-\frac{i}{t} \int_{i}^{z_{S_{t}}} \omega^{\xi}\right)\right],
\end{aligned}
$$

where $S_{t}$ denotes the hitting time of the line of equation $y=e^{-t}$ by the Brownian motion on $H$ starting at $i$.

Proof. Using the invariance of the Liouville measure under the action of $\theta_{t}$ and performing first the change of variables $\xi \theta_{t} \rightarrow \xi$, and then change $s-t$ into $s$, the left hand side becomes

$$
\int m(d \xi) \exp \left(\frac{i}{t} \int_{-t}^{0} \phi\left(\xi \theta_{s}\right) d s\right)
$$


With that remark we do not have to consider $\int_{i}^{i e^{t}} \omega^{\xi}$ anymore, but $-\int_{i}^{i e^{-t}} \omega^{\xi}$.

By the invariance of $m$ under the right action of $\theta_{u}^{+}$, we get

$$
\int m(d \xi) \exp \left(\frac{i}{t} \int_{-t}^{0} \phi\left(\xi \theta_{s}\right) d s\right)=\int m(d \xi) \exp \left(\frac{i}{t} \int_{-t}^{0} \phi\left(\xi \theta_{u}^{+} \theta_{s}\right) d s\right)
$$

for all $u \in \mathbb{R}$, thus

$$
\begin{aligned}
\int m(d \xi) \exp \left(\frac{-i}{t}\right. & \left.\int_{0}^{-t} \phi\left(\xi \theta_{s}\right) d s\right) \\
& =\iint \nu_{t}(d u) m(d \xi) \exp \left(\frac{-i}{t} \int_{0}^{-t} \phi\left(\xi \theta_{u}^{+} \theta_{s}\right) d s\right)
\end{aligned}
$$

where $\nu_{t}(d u)$ is any probability measure on $\mathbb{R}$.

But from Section $3, \theta_{u}^{+} \theta_{t}=T_{u+i e^{t}}$, hence

$$
\int_{0}^{-t} \phi\left(\xi \theta_{u}^{+} \theta_{s}\right) d s=\int_{u+i}^{u+i e^{-t}} \omega^{\xi}
$$

We have now to study

$$
\begin{aligned}
& \iint \nu_{t}(d u) m(d \xi) \exp \left(\frac{-i}{t} \int_{u+i}^{u+i e^{-t}} \omega^{\xi}\right) \\
& =\iint \nu_{t}(d u) m(d \xi) \exp \left(\frac{-i}{t} \int_{i}^{u+i e^{-t}} \omega^{\xi}\right) \\
& \quad-\iint \nu_{t}(d u) m(d \xi) \exp \left(\frac{-i}{t} \int_{i}^{u+i e^{-t}} \omega^{\xi}\right)\left(1-\exp \left(\frac{i}{t} \int_{i}^{u+i} \omega^{\xi}\right)\right) .
\end{aligned}
$$

We are now choosing for $\nu_{t}$ the Cauchy law with parameter $1-e^{-t}$, namely the hitting distribution of the line $y=e^{-t}$ by the Brownian motion. The last term vanishes as $t$ goes to $+\infty$, by dominated convergence since

$$
\frac{\nu_{t}(d u)}{d u}=\frac{1-e^{-t}}{\left(1-e^{-t}\right)^{2}+u^{2}} \leq \frac{1}{\frac{1}{4}+u^{2}}, \quad \text { for } t \geq \log 2
$$


With this choice of $\nu_{t}$,

$$
\begin{aligned}
& \iint \nu_{t}(d u) m(d \xi) \exp \left(\frac{-i}{t} \int_{i}^{u+i e^{-t}}\right.\left.\omega^{\xi}\right) \\
&=E\left[\int m(d \xi) \exp \left(\frac{-i}{t} \int_{i}^{z_{S_{t}}} \omega^{\xi}\right)\right]
\end{aligned}
$$

which can also be written,

$$
E\left[\int m(d \xi) \exp \left(\frac{-i}{t} \int_{0}^{S_{t}}\left\langle\omega^{\xi}, \circ d z_{s}\right\rangle\right)\right],
$$

where $\circ$ denotes the Stratonovich integral as in [6]. It is indeed the stochastic integral for which the differential calculus coincides with the usual one; in other words, if

$$
F(z)=\int_{i}^{z} \omega^{\xi}, \quad F\left(z_{t}\right)=\int_{0}^{t}\left\langle\omega^{\xi}, \circ d z_{s}\right\rangle
$$

\section{From Stratonovich to Itô.}

By previous lemma, we have to study

$$
\lim _{t \rightarrow+\infty} E\left[\int m(d \xi) \exp \left(\frac{-i}{t} \int_{0}^{S_{t}}\left\langle\omega^{\xi}, \circ d z_{s}\right\rangle\right)\right]
$$

The difficulty lies in the fact that $\omega^{\xi}$ is not a priori harmonic, and so the integral $\int_{0}^{t}\left\langle\omega^{\xi}, \circ d z_{s}\right\rangle$ is not a martingale, so that we cannot directly treat the problem using excursion theory as it was done in [10].

Let us examine the integral (we denote $\xi_{s}=\xi T_{z_{s}}$ )

$$
\begin{aligned}
\int_{0}^{t}\left\langle\omega^{\xi}, \circ d z_{s}\right\rangle= & \int_{0}^{t}\left(\frac{\phi\left(\xi T_{z_{s}}\right)}{y_{s}} \circ d y_{s}+\frac{\tilde{\phi}\left(\xi T_{z_{s}}\right)}{y_{s}} \circ d x_{s}\right) \\
= & \int_{0}^{t}\left(\phi\left(\xi_{s}\right) d W_{s}^{(2)}+\tilde{\phi}\left(\xi_{s}\right) d W_{s}^{(1)}\right) \\
& +\frac{1}{2} \int_{0}^{t}\left(d\left\langle\frac{\phi\left(\xi T_{z_{s}}\right)}{y_{s}}, y_{s}\right\rangle+d\left\langle\frac{\tilde{\phi}\left(\xi T_{z_{s}}\right)}{y_{s}}, x_{s}\right\rangle\right) .
\end{aligned}
$$


By Itô's formula,

$$
\begin{aligned}
d\left\langle\frac{\phi\left(\xi T_{z_{s}}\right)}{y_{s}}, y_{s}\right\rangle & =\frac{\partial}{\partial y}\left(\frac{\phi\left(\xi T_{z}\right)}{y}\right) d\left\langle y_{s}, y_{s}\right\rangle \\
& =\left(2 y_{s} \frac{\partial \phi}{\partial y}\left(\xi T_{z_{s}}\right)-2 \phi\left(\xi T_{z_{s}}\right)\right) d s
\end{aligned}
$$

Similarly,

$$
d\left\langle\frac{\phi\left(\xi T_{z_{s}}\right)}{y_{s}}, x_{s}\right\rangle=\frac{\partial}{\partial x}\left(\frac{\tilde{\phi}\left(\xi T_{z}\right)}{y}\right) d\left\langle x_{s}, x_{s}\right\rangle=\left(2 y_{s} \frac{\partial \tilde{\phi}}{\partial x}\left(\xi T_{z_{s}}\right)\right) d s .
$$

Thus

$$
\begin{aligned}
\int_{0}^{t}\left\langle\omega^{\xi}, \circ d z_{s}\right\rangle= & \int_{0}^{t} \phi\left(\xi_{s}\right) d W_{s}^{(2)}+\tilde{\phi}\left(\xi_{s}\right) d W_{s}^{(1)} \\
& +\int_{0}^{t}\left(y_{s} \frac{\partial \phi}{\partial y}\left(\xi T_{z_{s}}\right)+y_{s} \frac{\partial \tilde{\phi}}{\partial x}\left(\xi T_{z_{s}}\right)-\phi\left(\xi T_{z_{s}}\right)\right) d s
\end{aligned}
$$

which can also be written

$$
\begin{aligned}
\int_{0}^{t}\left\langle\omega^{\xi}, \circ d z_{s}\right\rangle= & \int_{0}^{t} \phi\left(\xi_{s}\right) d W_{s}^{(2)}+\tilde{\phi}\left(\xi_{s}\right) d W_{s}^{(1)} \\
& +\int_{0}^{t}\left(L_{0} \phi+L_{+} \tilde{\phi}-\phi\right)\left(\xi T_{z_{s}}\right) d s .
\end{aligned}
$$

We notice that the last term describes the "lack of harmonicity" of the form $\omega^{\xi}$. Indeed $\left(L_{0} \phi+L_{+} \tilde{\phi}-\phi=0\right)$ as soon as $\omega^{\xi}$ is harmonic and we can then see that $\int_{0}^{t}\left\langle\omega^{\xi}, \circ d z_{s}\right\rangle$ is a martingale.

We show that the second term has no influence on the limit by the ergodic theorem, proving that $L_{0} \phi+L_{+} \tilde{\phi}-\phi$ is in $L^{1}(m)$, and that its mean value is equal to 0 . For that purpose we shall prove two lemmas:

Lemma 7. With the notations of Section $4, L_{+} \tilde{\phi}^{\prime}=-L_{0} \phi+\phi$.

ProOF. By Lemma 2, we have just to check the following equality

$$
\begin{aligned}
\left(y_{i} \sin \theta_{i} \frac{\partial}{\partial y_{i}}+(1\right. & \left.\left.-\cos \theta_{i}\right) \frac{\partial}{\partial \theta_{i}}\right)\left(u\left(\frac{y_{i}}{h_{i}}\right) y_{i} \cos \theta_{i}\right) \\
= & -\left(y_{i} \cos \theta_{i} \frac{\partial}{\partial y_{i}}+\sin \theta_{i} \frac{\partial}{\partial \theta_{i}}\right)\left(-u\left(\frac{y_{i}}{h_{i}}\right) y_{i} \sin \theta_{i}\right) \\
& -u\left(\frac{y_{i}}{h_{i}}\right) y_{i} \sin \theta_{i} .
\end{aligned}
$$


REMARK. $\tilde{\phi}^{\prime}$ has the property to make coclosed the form

$$
\tilde{\phi}^{\prime}\left(\xi T_{z}\right) \frac{d x}{y}+\phi\left(\xi T_{z}\right) \frac{d x}{y} .
$$

Lemma 8. $f=L_{0} \phi+L_{+} \tilde{\phi}-\phi$, is m-integrable.

Proof. By lemmas 6 and 7 , it is enough to prove that $L_{+}\left(\tilde{\phi}-\tilde{\phi}^{\prime}\right)$ is integrable on $\pi^{-1}\left(V_{i}\right)$.

Set for $\xi \in \pi^{-1}\left(V_{i}\right)$

$$
\left(\begin{array}{cc}
a_{i} & b_{i} \\
c_{i} & d_{i}
\end{array}\right)=\gamma_{i} g_{i}(\xi)
$$

Note that $c_{i}^{2}+d_{i}^{2} \leq 3 / h_{i}$. Then $\tilde{\phi}(\xi)=-R_{1} L_{+} \phi(\xi)$ can be written

$$
\begin{aligned}
\tilde{\phi}(\xi)= & -\int_{0}^{+\infty} e^{-s} L_{+} \phi\left(\xi \theta_{s}\right) d s \\
= & -\int_{0}^{\log \left(2 /\left(c_{i}^{2} h_{i}\right)\right)} e^{-s} L_{+} \phi\left(\xi \theta_{s}\right) d s-\int_{\left.\log \left(2 / c_{i}^{2} h_{i}\right)\right)}^{+\infty} e^{-s} L_{+} \phi\left(\xi \theta_{s}\right) d s \\
= & -\frac{\lambda_{i}}{X_{i}} \int_{0}^{\log \left(2 /\left(c_{i}^{2} h_{i}\right)\right)}\left(\frac{2 c_{i}^{2} e^{2 s}}{\left(d_{i}^{2}+c_{i}^{2} e^{2 s}\right)^{2}}-\frac{8 c_{i}^{2} d_{i}^{2} e^{2 s}}{\left(d_{i}^{2}+c_{i}^{2} e^{2 s}\right)^{3}}\right) d s \\
& -\int_{\left.\log \left(2 / c_{i}^{2} h_{i}\right)\right)}^{+\infty} e^{-s} L_{+} \phi\left(\xi \theta_{s}\right) d s .
\end{aligned}
$$

Since in matricial coordinates

$$
\begin{gathered}
L_{+} \phi(\xi)=\frac{\lambda_{i}}{X_{i}}\left(\frac{2 c_{i}^{2}}{\left(c_{i}^{2}+d_{i}^{2}\right)^{2}}-\frac{8 c_{i}^{2} d_{i}^{2}}{\left(c_{i}^{2}+d_{i}^{2}\right)^{3}}\right), \\
\tilde{\phi}(\xi)=-\frac{\lambda_{i}}{X_{i}}\left[\frac{d_{i}^{2}-c_{i}^{2} x}{\left(d_{i}^{2}+c_{i}^{2} x\right)^{2}}\right]_{1}^{2 / c_{i}^{2} h_{i}}+\frac{h_{i} c_{i}^{2}}{2} \tilde{\phi}\left(\xi T_{2 i /\left(c_{i}^{2} h_{i}\right)}\right) \\
=\tilde{\phi}^{\prime}(\xi)+\frac{\lambda_{i}}{X_{i}} \frac{\frac{2}{h_{i}}-d_{i}^{2}}{\left(\frac{4}{h_{i}^{2}}+d_{i}^{2}\right)^{2}}+\frac{h_{i} c_{i}^{2}}{2} \tilde{\phi}\left(\xi T_{2 i /\left(c_{i}^{2} h_{i}\right)}\right) .
\end{gathered}
$$

It follows that $L_{+}\left(\tilde{\phi}-\tilde{\phi}^{\prime}\right)$ can be decomposed in the sum of two terms, which both appear to be bounded. 
The first one is

$$
\begin{aligned}
L_{+}\left(\frac{\frac{2}{h_{i}}-d_{i}^{2}}{\left(\frac{4}{h_{i}^{2}}+d_{i}^{2}\right)^{2}}\right) & =\left(a_{i} \frac{\partial}{\partial b_{i}}+c_{i} \frac{\partial}{\partial d_{i}}\right)\left(\frac{\frac{2}{h_{i}}-d_{i}^{2}}{\left(\frac{4}{h_{i}^{2}}+d_{i}^{2}\right)^{2}}\right) \\
& =-\frac{\frac{8 c_{i} d_{i}}{h_{i}}}{\left(\frac{4}{h_{i}^{2}}+d_{i}^{2}\right)^{3}}-\frac{2 c_{i} d_{i}}{\left(\frac{4}{h_{i}^{2}}+d_{i}^{2}\right)^{2}},
\end{aligned}
$$

which is clearly bounded since $\left|c_{i}\right|$ and $\left|d_{i}\right|$ are bounded by $1 / \sqrt{h}_{i}$.

The second one is $L_{+}(\psi)$ with

$$
\psi(\xi)=\frac{h_{i} c_{i}^{2}}{2} \tilde{\phi}\left(\xi T_{2 i /\left(c_{i}^{2} h_{i}\right)}\right)
$$

Note that for that $z$ close to $i$,

$$
\psi\left(\xi T_{z}\right)=\frac{h_{i} c_{i}^{2} y}{2} \tilde{\phi}\left(\xi T_{z} T_{2 i /\left(c_{i}^{2} y h_{i}\right)}\right)=\frac{h_{i} c_{i}^{2} y}{2} \tilde{\phi}\left(\xi T_{x+2 i /\left(c_{i}^{2} h_{i}\right)}\right)
$$

and therefore

$$
\begin{aligned}
L_{+} \psi\left(\xi T_{z}\right) & =y \frac{\partial \psi\left(\xi T_{z}\right)}{\partial x} \\
& =\frac{c_{i}^{4} h_{i}^{2}}{4} y^{2}\left(\frac{2}{h_{i} c_{i}^{2}} \frac{\partial}{\partial x} \tilde{\phi}\left(\xi T_{x+2 i /\left(c_{i}^{2} h_{i}\right)}\right)\right) \\
& =\frac{c_{i}^{4} h_{i}^{2}}{4} y^{2}\left(L_{+} \tilde{\phi}\right)\left(\xi T_{x+2 i /\left(c_{i}^{2} h_{i}\right)}\right) .
\end{aligned}
$$

Hence

$$
L_{+} \psi(\xi)=\frac{c_{i}^{4} h_{i}^{2}}{4}\left(L_{+} \tilde{\phi}\right)\left(\xi T_{2 i /\left(c_{i}^{2} h_{i}\right)}\right),
$$

$c_{i}^{4} h_{i}^{2} / 4$ is clearly bounded, moreover as shown in the proof of Lemma $7, \xi T_{2 i /\left(c_{i}^{2} h_{i}\right)}$ belongs to $V_{i} \backslash W_{i}$, which is relatively compact and $L_{+} \tilde{\phi}$ is continuous. The integrability of $f$ on $T^{1} M$ is now proven.

We can now state:

Lemma 9. The integral of $f$ on $T^{1} M$ vanishes. 
Proof. From Lemma 7 it is enough to show that

$$
\int_{T^{1} M} L_{+}\left(\tilde{\phi}-\tilde{\phi}^{\prime}\right)(\xi) m(d \xi)=0
$$

Let $g_{n}^{0}$ a sequence of smooth positive functions on $M$, increasing towards 1 as $\mathrm{n}$ goes to $\infty$, and such that $\left\|\nabla g_{n}^{0}\right\|_{\infty}$ is less than some constant $C$ for all $n$. Set $g_{n}=g_{n}^{0} \circ \pi$. An integration by part yields

$$
\int_{T^{1} M} g_{n} L_{+}\left(\tilde{\phi}-\tilde{\phi}^{\prime}\right)(\xi) m(d \xi)=\int_{T^{1} M}\left(\tilde{\phi}^{\prime}-\tilde{\phi}\right) L_{+} g_{n}(\xi) m(d \xi)
$$

and the result follows by dominated convergence, letting $n$ increase to infinity.

Hence, we reduced our problem to the study of

$$
\lim _{t \rightarrow+\infty} E\left[\int m(d \xi) \exp \left(\frac{-i \sqrt{2}}{t} \int_{0}^{S_{t}} \tilde{\phi}\left(\xi_{s}\right) d W_{s}^{(1)}+\phi\left(\xi_{s}\right) d W_{s}^{(2)}\right)\right] .
$$

\section{Calculation of the limit via excursion theory.}

Lemma 10. $S_{t} / t$ converges almost surely towards 1 as $t \longrightarrow+\infty$.

Proof. Since

$$
y_{t}=\exp \left(\sqrt{2} W_{t}^{(1)}-t\right), \quad \text { for } t \geq 0,
$$

we have

$$
S_{t}-t=\sqrt{2} W_{S_{t}}^{(1)}
$$

So the graph of $t \longmapsto S_{t}$ is symmetric to the graph of $t \longmapsto t-\sqrt{2} W_{t}^{(1)}$, with respect to the first diagonal and

$$
\frac{t-\sqrt{2} W_{t}^{(1)}}{t} \longrightarrow 1, \quad \text { almost surely, as } t \rightarrow \infty
$$

Set

$$
\frac{N_{t, 1}}{t}=\frac{1}{t} \int_{0}^{t} \tilde{\phi}\left(\xi_{s}\right) \mathbf{1}_{\left\{\pi\left(\xi_{s}\right) \notin W\right\}} d W_{s}^{(1)}+\phi\left(\xi_{s}\right) \mathbf{1}_{\left\{\pi\left(\xi_{s}\right) \notin W\right\}} d W_{s}^{(2)}
$$


Lemma 11. $N_{S_{t}, 1} / t$ converges to 0 in $L^{2}$.

Proof. $N_{t, 1}$ is a martingale with bracket

$$
\int_{0}^{t}\left(\tilde{\phi}^{2}\left(\xi_{s}\right) \mathbf{1}_{\left\{\pi\left(\xi_{s}\right) \notin W\right\}}+\phi^{2}\left(\xi_{s}\right) \mathbf{1}_{\left\{\pi\left(\xi_{s}\right) \notin W\right\}}\right) d s .
$$

Since $\tilde{\phi}$ and $\phi$ are bounded on $\pi^{-1}\left(W^{c}\right)$, say by $K$, for all integer $M$,

$$
E\left[N_{S_{t} \wedge M}^{2}\right]=E\left[\int_{0}^{S_{t} \wedge M}\left(\tilde{\phi}^{2}\left(\xi_{s}\right) \mathbf{1}_{\left\{\pi\left(\xi_{s}\right) \notin W\right\}}+\phi^{2}\left(\xi_{s}\right) \mathbf{1}_{\left\{\pi\left(\xi_{s}\right) \notin W\right\}}\right) d s\right]
$$

so that

$$
E\left[N_{S_{t} \wedge M}^{2}\right] \leq K^{2} E\left[S_{t} \wedge M\right]
$$

But $S_{t} \wedge M+\log \left(y_{S_{t} \wedge M}\right)=2 W_{S_{t} \wedge M}^{(1)}$, and as far as $\log \left(y_{S_{t} \wedge M}\right) \geq-t$,

$$
E\left[S_{t} \wedge M\right] \leq t
$$

so that by Fatou's lemma, we get when $M$ converges to $\infty$,

$$
E\left[N_{S_{t}}^{2}\right] \leq K^{2} t
$$

and we deduce the lemma.

Set now,

$$
\frac{N_{S_{t}, 2}}{t}=\frac{1}{t} \int_{0}^{S_{t}} \tilde{\phi}\left(\xi_{s}\right) \mathbf{1}_{\left\{\pi\left(\xi_{s}\right) \in W\right\}} d W_{s}^{(1)}+\phi\left(\xi_{s}\right) \mathbf{1}_{\left\{\pi\left(\xi_{s}\right) \in W\right\}} d W_{s}^{(2)}
$$

\section{Lemma 12.}

$$
\frac{1}{t} \int_{0}^{S_{t}}\left(\tilde{\phi}\left(\xi_{s}\right)-\tilde{\phi}^{\prime}\left(\xi_{s}\right)\right) \mathbf{1}_{\left\{\pi\left(\xi_{s}\right) \in W\right\}} d W_{s}^{(1)}
$$

converges to 0 in $L^{2}$.

Proof. The same proof as in the previous lemma yields the result, since $\tilde{\phi}-\tilde{\phi}^{\prime}$ is easily seen to be bounded.

The averaged integral in the limit can therefore be replaced by

$$
\frac{\sqrt{2}}{t} \int_{0}^{S_{t}} \tilde{\phi}^{\prime}\left(\xi_{s}\right) \mathbf{1}_{\left\{\pi\left(\xi_{s}\right) \in W\right\}} d W_{s}^{(1)}+\phi\left(\xi_{s}\right) \mathbf{1}_{\left\{\pi\left(\xi_{s}\right) \in W\right\}} d W_{s}^{(2)} .
$$


In order to use excursion theory, we have to get rid of "incomplete excursions" containing 0 and $S_{t}$. For that purpose we introduce $T_{\xi}$ the first exit time of $\pi^{-1}(W)$ of the Brownian motion starting at $\xi$, and $S_{t}^{\xi}$ its first exit time of $\pi^{-1}(W)$ after $S_{t}$. (N.B. $T_{\xi}$ vanishes if $\pi(\xi) \notin W$ and $T_{t}^{\xi}=S_{t}$ when $\left.\pi\left(\xi_{S_{t}}\right) \notin W\right)$.

Note that under $m \otimes \mathbb{P}$, the distributions of

$$
\int_{0}^{T^{\xi}} \tilde{\phi}^{\prime}\left(\xi_{s}\right) \mathbf{1}_{\left\{\pi\left(\xi_{s}\right) \in W\right\}} d W_{s}^{(1)}+\phi\left(\xi_{s}\right) \mathbf{1}_{\left\{\pi\left(\xi_{s}\right) \in W\right\}} d W_{s}^{(2)}
$$

and

$$
\int_{S_{t}}^{S_{t}^{\xi}} \tilde{\phi}^{\prime}\left(\xi_{s}\right) \mathbf{1}_{\left\{\pi\left(\xi_{s}\right) \in W\right\}} d W_{s}^{(1)}+\phi\left(\xi_{s}\right) \mathbf{1}_{\left\{\pi\left(\xi_{s}\right) \in W\right\}} d W_{s}^{(2)}
$$

are independent of $t$ ( for the second integral, this follows from the $T_{z^{-}}$ invariance of $m$ and the independence of $\xi$ and $S_{t}$ ). Their quotients by $t$ converge therefore to zero in probability. The averaged integral in the limit can finally be replaced by (Lemma 3),

$$
\begin{aligned}
H_{t}^{\xi} & =\frac{\sqrt{2}}{t} \int_{T^{\xi}}^{S_{t}^{\xi}} \tilde{\phi}^{\prime}\left(\xi_{s}\right) \mathbf{1}_{\left\{\pi\left(\xi_{s}\right) \in W\right\}} d W_{s}^{(1)}+\phi\left(\xi_{s}\right) \mathbf{1}_{\left\{\pi\left(\xi_{s}\right) \in W\right\}} d W_{s}^{(2)} \\
& =\frac{1}{t} \int_{T^{\xi}}^{S_{t}^{\xi}} \sum_{i=1}^{n} \frac{\lambda_{i}}{X_{i}}\left\langle s_{i}^{\star} \gamma_{i}^{\star}(d x) \mathbf{1}_{\left\{z_{s}^{\xi} \in W_{i}\right\}}, \circ d z_{s}^{\xi}\right\rangle,
\end{aligned}
$$

where $z_{s}^{\xi}=\pi\left(\xi_{s}\right)$ is the Brownian motion on $\Gamma \backslash H$, starting from $\pi(\xi)$.

We now denote by $E$ the expected value with respect to $m \otimes P$. Denote $e_{i}^{\xi}$ the excursions of $z_{s}^{\xi}$ in $W_{i}$, and $\hat{e}_{i}^{\xi}$ its lift into $H$, starting from the image in $\gamma_{i} \mathcal{F}_{i}$ of the starting point of $e_{i}^{\xi}$. Denote $a\left(\hat{e}_{i}^{\xi}\right)$ and $b\left(\hat{e}_{i}^{\xi}\right)$ the starting point and the endpoint of $\hat{e}_{i}^{\xi}$ in $\mathrm{H}$ and denote $\left[S\left(e_{i}^{\xi}\right), T\left(e_{i}^{\xi}\right)\right]$ the corresponding time interval.

With these notations,

$$
H_{t}^{\xi}(\omega)=\frac{1}{t} \sum_{i=1}^{n} \frac{\lambda_{i}}{X_{i}}\left(\sum_{\substack{e_{i}^{\xi} \\ 0<S\left(e_{i}^{\xi}\right) \leq S_{t}}} b\left(\hat{e}_{i}^{\xi}\right)-a\left(\hat{e}_{i}^{\xi}\right)\right) .
$$

From excursion theory we get that

$$
\left.E\left[\exp \left(i H_{t}^{\xi}(\omega)\right)\right]=E\left[\exp \left(\sum_{i=1}^{n} \hat{E}_{h_{i}}\left(\exp \left(i \frac{\lambda_{i}}{X_{i}} \frac{X}{t}\right)-1\right)\right) L_{i, S_{t}}\right)\right],
$$


where $L_{i, t}$ is the value at time $t$ of a local time on $\partial V_{i}$ of $z_{s}^{\xi}$ and $\hat{E}_{h_{i}}$ is the excursion law of the Brownian motion on $\mathrm{H}$, above the line $y=h_{i}$. Its normalization depends on the choice of $L_{i}$, via the identity

$$
E\left[\frac{1}{t} \int_{0}^{t} \mathbf{1}_{\left\{z_{s}^{\xi} \in W_{i}\right\}} d s\right]=E\left[\frac{L_{i, t}}{t} \hat{E}_{h_{i}}(\zeta)\right]
$$

( $\zeta$ being the excursion lifetime).

$X$ is the abscissa of the excursion endpoint.

By definition of $\hat{E}_{h_{i}}$,

$$
\begin{aligned}
& \left.E\left[\exp \left(\sum_{i=1}^{n} \hat{E}_{h_{i}}\left(\exp \left(i \frac{\lambda_{i}}{X_{i}} \frac{X}{t}\right)-1\right)\right) L_{i, S_{t}}\right)\right] \\
& =E\left[\exp \left(\sum_{i=1}^{n} \lim _{\varepsilon \rightarrow 0} \frac{1}{K \varepsilon} E_{x, h_{i}(1+\varepsilon)}\left(\exp \left(i \frac{\lambda_{i}}{t X_{i}}\left(x_{\tau_{h_{i}}}-x\right)-1\right)\right) L_{i, S_{t}}\right)\right]
\end{aligned}
$$

where $\tau_{h_{i}}$ denotes the hitting time of the line $y=h_{i}$ by the Brownian motion on $H$ starting from the point $\left(x, h_{i}(1+\varepsilon)\right)$ and $K$ is a normalization constant related to the normalization of $L_{i}$.

This last expression equals

$$
\begin{aligned}
E\left[\operatorname { e x p } \left(\sum_{i=1}^{n} \lim _{\varepsilon \rightarrow 0} \frac{1}{K \varepsilon}\right.\right. & \left.E_{x, h_{i}(1+\varepsilon)}\left(\left(\exp \left(-\frac{\lambda^{2}}{t^{2} X_{i}^{2}} \int_{0}^{\tau_{h_{i}}} y_{s}^{2} d s\right)-1\right) L_{i, S_{t}}\right)\right] \\
& =E\left[\exp \left(\sum_{i=1}^{n} \lim _{\varepsilon \rightarrow 0} \frac{1}{K \varepsilon}\left(\frac{\phi_{i}\left((1+\varepsilon) h_{i}\right)}{\phi_{i}\left(h_{i}\right)}-1\right) L_{i, S_{t}}\right)\right] \\
& =E\left[\exp \left(\sum_{i=1}^{n} \frac{h_{i}}{K}\left(\log \phi_{i}\right)^{\prime}\left(h_{i}\right) L_{i, S_{t}}\right)\right]
\end{aligned}
$$

where by the Feynman-Kac formula, $\phi_{i}$ solves the differential equation

$$
y^{2} \phi_{i}^{\prime \prime}-\frac{\lambda_{i}^{2}}{t^{2} X_{i}^{2}} y^{2} \phi_{i}=0
$$

with $\phi_{i}\left(h_{i}\right)=1$ and $\phi_{i}$ bounded at $+\infty$. Therefore

$$
\phi_{i}(y)=\exp \left(-\frac{\left|\lambda_{i}\right|}{t X_{i}}\left(y-h_{i}\right)\right)
$$


and our expression takes the form

$$
E\left[\exp \left(-\sum_{i=1}^{n} \frac{h_{i}\left|\lambda_{i}\right|}{K t X_{i}} L_{i, S_{t}}\right)\right] .
$$

We now come back to the problem of normalizations. If $\hat{E}_{h_{i}}$ is normalized in such a way that $\hat{E}_{h_{i}}(\zeta)=1$, we have

$$
E\left[\frac{1}{t} \int_{0}^{t} \mathbf{1}_{\left\{z_{s}^{\xi} \in W_{i}\right\}} d s\right]=\frac{E\left[L_{i, t}\right]}{t}
$$

Since under $m \otimes P_{\xi}, z_{s}$ is an ergodic process with invariant measure $d x d y /|M| y^{2}$,

$$
\frac{E\left[L_{i, t}\right]}{t}=\frac{1}{|M|} \int_{V_{i}} \frac{d x d y}{y^{2}}=\frac{X_{i}}{|M| h_{i}} .
$$

The ergodic theorem for additive functionals (e.g. [14]) yields the almost sure convergence of $L_{i, t} / t$ towards $X_{i} /\left(|M| h_{i}\right)$. As $S_{t} / t \longrightarrow 1$, $L_{i, S_{t}} / t$ converges also, almost surely, towards $X_{i} /\left(|M| h_{i}\right)$.

The expectation of the excursion lifetime equals

$$
\lim _{\varepsilon \rightarrow 0} \frac{1}{K \varepsilon} E_{h_{i}(1+\varepsilon)}\left[\tau_{h_{i}}\right]=\lim _{\varepsilon \rightarrow 0} \lim _{\alpha \rightarrow 0}-\frac{1}{K \varepsilon \alpha} E_{h_{i}(1+\varepsilon)}\left[\exp \left(-\alpha \tau_{h_{i}}\right)-1\right],
$$

by monotone convergence (monotonicity in $\alpha$ follows from the convexity of the exponential).

The normalization of the excursion lifetime yields

$$
1=\lim _{\varepsilon \rightarrow 0} \lim _{\alpha \rightarrow 0}-\frac{1}{K \varepsilon \alpha}\left(\psi_{i, \alpha}\left(h_{i}(1+\varepsilon)\right)-1\right),
$$

where $\psi_{i, \alpha}$ is the solution of the differential equation

$$
y^{2} \psi_{i, \alpha}^{\prime \prime}(y)-\alpha \psi_{i, \alpha}(y)=0
$$

bounded at $\infty$ and such that $\psi_{i, \alpha}\left(h_{i}\right)=1$.

Hence $\psi_{i, \alpha}(y)=\left(y / h_{i}\right)^{\mu}$ where $\mu$ is the negative root of the equation $\mu(\mu-1)-\alpha=0$, namely

$$
\mu=\frac{1}{2}(1-\sqrt{1+4 \alpha}),
$$


therefore

$$
\lim _{\varepsilon \rightarrow 0} \lim _{\alpha \rightarrow 0} \frac{(1+\varepsilon)^{\mu}-1}{\varepsilon \alpha}=1 \quad \text { and } \quad K=\lim _{\alpha \rightarrow 0}-\frac{\mu}{\alpha}=1 .
$$

Finally

$$
\lim _{t \rightarrow \infty} E\left[\exp \left(-\sum_{i=1}^{n} \frac{h_{i}\left|\lambda_{i}\right|}{K t X_{i}} L_{i, S_{t}}\right)\right]=\exp \left(-\sum_{i=1}^{n} \frac{\left|\lambda_{i}\right|}{|M|}\right) .
$$

Hence

$\lim _{t \rightarrow \infty} E\left(\exp \left(\sum_{i=1}^{n} \hat{E}_{h_{i}}\left(\exp \left(i \frac{\lambda_{i}}{X_{i}} \frac{X}{t}\right)-1\right) L_{i, S_{t}}\right)\right]=\exp \left(-\sum_{i=1}^{n} \frac{\left|\lambda_{i}\right|}{|M|}\right)$

and the average on $T^{1} M$ of $\exp \left(i H_{t}^{\xi}\right)$, converges towards

$$
\exp \left(-\sum \frac{\left|\lambda_{i}\right|}{|M|}\right)
$$

which ends the proof of Theorem 1.

\section{References.}

[1] Franchi, J., Théorème des résidus asymptotique pour le mouvement brownien sur une surface riemanienne compacte. Ann. Inst. H. Poincaré, Probabilités et Statistiques 27 (1991), 445-462.

[2] Franchi, J., Le Jan, Y., Brownian charges around loops. Probab. Theory Relat. Fields. 104 (1996), 501-514.

[3] Guivarc'h, Y., Le Jan, Y., Sur l'enroulement du flot géodésique. $C$. $R$. Acad. Sci. Paris. 311 Série I (1990), 645-648.

[4] Guivarc'h, Y., Le Jan, Y., Asymptotic winding of the geodesic flow on modular surfaces and continuous fractions. Ann. Sci. Ecole Norm. Sup. 26 Série IV (1993), 23-50.

[5] Hopf, E., Ergodicity theory and the geodesic flow on surfaces of constant negative curvature. Bull. Amer. Math. Soc. 77 (1971), 863-877.

[6] Ikeda, N., Watanabe, S., Stochastic differential equations and diffusion processes. North Holland, 1981.

[7] Ledrappier, F., Central limit theorem in negative curvature. Ann. Probab. 23 (1995), 1219-1233. 
[8] Ledrappier, F., Harmonic 1-forms on the stable foliation. Bol. Soc. Bras. Mat. 25 (1995), 121-138.

[9] Le Gall, J-F., Yor, M., Enlacements du mouvement brownien autour des courbes de l'espace. Trans. Amer. Math. Soc. 317 (1990), 687-722.

[10] Le Jan, Y., Sur l'enroulement géodésique des surfaces de Riemann. C. R. Acad. Sci. Paris 314 (1992), 763-765.

[11] Le Jan, Y., The central limit theorem for the geodesic flow on non compact manifolds of constant negative curvature. Duke Math. J. $\mathbf{7 4}$ (1994), 159-175.

[12] Lyons, T., Mc Kean, H. P., Windings of the plane Brownian motion. Advances in Math. 51 (1984), 212-225.

[13] Ratner, M., The central limit theorem for geodesic flows on $\mathrm{N}$-dimensional manifolds of negative curvature. Israel J. of Mat. 16 (1973), 180197.

[14] Revuz, D., Yor, M., Continuous martingales and Brownian motion. Springer Verlag, 1991.

[15] Sinaï, Y. G., The central limit theorem for geodesic flows on constant negative curvature. Dokl. Acad. Nauk SSSR 133 (1960), 1303-1306.

[16] Spitzer, F., Some theorems concerning 2-dimensional Brownian motion. Trans. Amer. Math. Soc. 87 (1958), 187-197.

[17] Sullivan, D., Disjoint spheres, approximation by imaginary quadratic numbers and the logarithm law for geodesics. Acta Math. 149 (1983), 123-237.

Recibido: 20 de mayo de 1.996

Revisado: 21 de abril de 1.997

Nathanaël Enriquez and Yves Le Jan

Départment de Mathématiques

Bât 425

Université Paris Sud

91405 Orsay

FRANCE

enriquez@proba . jussieu.fr

Yves.LeJan@math.u-psud.fr 